\title{
A proposed protocol for the management of COVID-19 in Egypt
}

\section{Authors:}

Khaled Sorour and Hadeer El-menshawy

Correspondence should be addressed to (khaled151026@bue.edu.eg)

\section{Dosing regimen backbone:}

\begin{tabular}{|c|c|}
\hline Drug & Dose \\
\hline Umifenovir (Arbidol) & $\begin{array}{c}\text { Children over 12 years \& Adults: 200 } \\
\text { mg QID for 5 days followed by 200 mg } \\
\text { BID on the following 5 days. }\end{array}$ \\
\hline Indomethacin (Indocin) & 50 mg QID for 10 days \\
\hline
\end{tabular}

Tests that could be performed:

\begin{tabular}{|c|}
\hline Serum electrolytes \\
\hline Vitamin D \\
\hline LDH - Troponin \\
\hline ESR - CRP \\
\hline APTT - Fibrinogen - PT - D-dimer \\
\hline Creatinine clearance \\
\hline SGOT \& SGPT \\
\hline Test for Albumin levels \\
\hline CBC - Ferritin \\
\hline Oxygen saturation \\
\hline
\end{tabular}


Priority should be given to:

* For all patients:

D-dimer, ESR, CBC, PT, Oxygen saturation (Assessed daily)

* For severely affected or worsening cases:

Serum Ferritin, Vitamin D, Serum electrolytes, Creatinine clearance, SGPT \& SGOT, LDH, cardiac Troponin-I, Albumin levels.

Other diagnostic tools:

- Chest CT scans to determine the level of lung injury or response to treatment. (For all patients)

- Echocardiography for severe cases to diagnose cardiac involvement or potential pulmonary embolism.

Add on therapy:

1- LMWH: $100 \mathrm{U} / \mathrm{kg} / 12 \mathrm{~h}$ for 3-5 days (a prophylactic anticoagulation for all patients experiencing 4-fold increase of D-dimer above normal upper limit.

Note:

- Patients that are refractory to LMWH or experiencing anti-thrombin-3 deficiency could be anti-coagulated with bivalirudin.

- The use of unfractionated heparin could be considered in COVID-19 patients with evidence of acute kidney injury (AKI).

- Patients who experience extreme elevation of D-dimer (more than 6-fold increase above normal upper limit) that have been refractory to $\mathrm{LMWH}$ therapy and are hemodynamically unstable with persistent hypoxemia and didn't meet the exclusion criteria are eligible for fibrinolytic therapy.

- A proposed approach was to administer $25 \mathrm{mg}$ of tPA over 2 hours followed by another dose infusion over the next 22 hours with a dose not exceeding $0.9 \mathrm{mg} / \mathrm{kg}$.

- In all COVID-19 patients on anticoagulant therapy, PT, APTT, fibrinogen and D-dimer tests should be frequently monitored and stoppage of therapy is assigned once returned to normal.

- Thrombocytopenia frequently reported with SARS-CoV-2 infection may prompt the discontinuation of the anti-coagulant therapy and initiation of platelet transfusion. 


\section{2- Maintaining oxygenation:}

- Alterations in breathing rate or oxygen saturation less than 93 should mandate Oxygen therapy since rapid deterioration and dropping of oxygen levels is observed in COVID-19.

- Humidified high flow nasal oxygen therapy could be considered and is superior to mechanical ventilation.

- A bag could be placed on the patient's head to prevent aerosol contamination.

\section{3- Other adjuncts:}

- ORS use could be considered daily to provide an immediate supply of necessary salts.

- Patients with profound pulmonary edema, could consider the use of furosemide while Hypoalbuminemia may mandate albumin transfusion to maintain fluid balance especially in those with hepatic decompensation.

- Maintaining a healthy diet that's rich in anti-oxidants and immunomodulatory agents is generally beneficial for patients during viral infections.

- Enteric route is highly encouraged to prevent bacterial translocation or stress- induced ulcer whenever accessible.

- Daily meals could incorporate olive oil, black cumin oil, chamomile and natural bee's honey. 


\section{References:}

Shike Geng, et al. (2020). "High flow nasal cannula is a good treatment option for COVID19." Heart \& Lung.

Gurka, D. P. and R. A. Balk (2008). "Acute Respiratory Failure." Critical Care Medicine: 773-794.

Moore, H. B., et al. (2020). "Is There a Role for Tissue Plasminogen Activator (tPA) as a Novel Treatment for Refractory COVID-19 Associated Acute Respiratory Distress Syndrome (ARDS)? ." J Trauma Acute Care Surg.

Lin, L., et al. (2020). "Hypothesis for potential pathogenesis of SARS-CoV-2 infection-a review of immune changes in patients with viral pneumonia." Emerg Microbes Infect 9(1): 727-732.

Zulfiqar, A. A., et al. (2020). "Immune Thrombocytopenic Purpura in a Patient with Covid19." N Engl J Med 382(18): e43.

Tianhong Xu, et al. (2020). Indomethacin has a potent antiviral activity against SARS CoV-2 in vitro and canine coronavirus in vivo. BioRxiv.

Zhu, Zhen et al. "Arbidol monotherapy is superior to lopinavir/ritonavir in treating COVID19." The Journal of infection, S0163-4453(20)30188-2. 10 Apr. 2020, doi:10.1016/j.jinf.2020.03.060

Ulasli, M., et al. (2014). "The effects of Nigella sativa (Ns), Anthemis hyalina (Ah) and Citrus sinensis (Cs) extracts on the replication of coronavirus and the expression of TRP genes family." Mol Biol Rep 41(3): 1703-1711.

Tang, N., et al. (2020). "Anticoagulant Treatment Is Associated With Decreased Mortality in Severe Coronavirus Disease 2019 Patients With Coagulopathy." J Thromb Haemost. 18(5).

Puertollano, M. A., et al. (2010). "[Olive oil, immune system and infection]." Nutr Hosp 25(1): 1-8.

Drummond, E. M., et al. (2013). "Inhibition of proinflammatory biomarkers in THP1 macrophages by polyphenols derived from chamomile, meadowsweet and willow bark." Phytother Res 27(4): 588-594.

Hashem, H. E. (2020). "IN Silico Approach of Some Selected Honey Constituents as SARSCoV-2 Main Protease (COVID-19) Inhibitors." Eurasian Journal of Medicine and Oncology 4(2).

Daneshkhah, A., et al, (2020). "The Possible Role of Vitamin D in Suppressing Cytokine Storm and Associated Mortality in COVID-19 Patients". MedRxiv. 